\title{
Sunitinib-suppressed miR-452-5p facilitates renal cancer cell invasion and metastasis through modulating SMAD4/SMAD7 signals
}

Wei Zhai ${ }^{1{ }^{*}}$ (D), Saiyang $\mathrm{Li}^{2+}$, Jin Zhang ${ }^{1}$, Yonghui Chen ${ }^{1}$, Junjie Ma², Wen Kong ${ }^{1}$, Dongkui Gong ${ }^{3}$, Junhua Zheng ${ }^{4}$, Wei Xue ${ }^{1 *}$ and Yunfei $X^{3^{*}}$

\begin{abstract}
Purpose: Although microRNAs (miRNAs) were revealed as crucial modulators in tumor metastasis and target therapy, our understanding of their roles in metastatic renal cell carcinoma (mRCC) and Sunitinib treatment was limited. Here we sought to identify human miRNAs that acted as key regulators in renal cancer metastasis and Sunitinib treatment.

Experimental design: We focused on 2 published microarray data to select out our anchored miRNA and then explored the roles of miR-452-5p both in vitro and in vivo, which was downregulated after Sunitinib treatment while upregulated in metastasis renal cell carcinoma (RCC) tissues.

Results: Here, we discovered that treating with Sunitinib, the targeted receptor tyrosine kinase inhibitor (TKI), inhibited renal cancer cell migration and invasion via attenuating the expression of miR-452-5p. The novel identified miR-452-5p was upregulated and associated with poor prognosis in RCC. Preclinical studies using multiple RCC cells and xenografts model illustrated that miR-452-5p could promote RCC cell migration and invasion in vitro and in vivo. Mechanistically, P65 could directly bind to the miR-452-5p promoter and thus transcriptionally induce miR452-5p expression, which led to post-transcriptionally abrogate SMAD4 expression, thus inhibition of its downstream gene SMAD7.
\end{abstract}

Conclusion: Our study presented a road map for targeting this newly identified miR-452-5p and its SMAD4/SMAD7 signals pathway, which imparted a new potential therapeutic strategy for mRCC treatment.

Keywords: Renal cell carcinoma, Sunitinib, miR-452-5p, P65, SMAD4, Metastasis

\section{Introduction}

Renal cell carcinoma was one of the most aggressive human urological malignancies, accounting for $2-3 \%$ of adult malignancies $[1,2]$. One third of patients had metastatic lesions detected at primary diagnosis, and $30 \%$ eventually developed metastatic renal cell carcinoma (mRCC) after surgery [3]. Because of its resistance to radiotherapy and chemo-therapy, surgical resection

\footnotetext{
*Correspondence: jacky_zw2002@hotmail.com; xuewei@renji.com; xuyunfeibb@sina.com

${ }^{\dagger}$ Wei Zhai and Saiyang Li contributed equally to this work.

${ }^{1}$ Department of Urology, Renji Hospital, School of Medicine in Shanghai Jiao Tong University, 160 Pujian Road, Pudong District, Shanghai 200127, China

${ }^{3}$ Department of Urology, Shanghai Tenth People's Hospital, School of

Medicine in Tongji University, Shanghai 200072, China

Full list of author information is available at the end of the article
}

remained the unique effective treatment $[4,5]$. Hence, a better understanding of the detailed mechanisms behind the pathogenesis of RCC and more effective treatment strategies were urgently required.

MicroRNAs (miRNAs), a group of small noncoding RNAs of about 20-24 nucleotides in length, negatively regulated gene expression [6, 7]. Accumulating evidence had demonstrated the involvement of miRNAs contributing to multiple metastatic steps in various human cancers $[8,9]$. Notably, plenty of miRNAs, such as miR-27a, miR-141 and miR-205 were reported to exert their roles in RCC cell invasion and metastasis [10-12]. In addition, our group already attested that miR-646 could suppress RCC cell migration and invasion [13]. Previous studies demonstrated that miR-452-5p acted

(C) The Author(s). 2018 Open Access This article is distributed under the terms of the Creative Commons Attribution 4.0 International License (http://creativecommons.org/licenses/by/4.0/), which permits unrestricted use, distribution, and 
as a tumor inducer or suppressor in multiple human cancers, involving osteosarcoma, breast cancer, non-small cell lung cancer, hepatocellular carcinoma and prostate cancer [14-18]. Nevertheless, whether miR-452-5p was implicated in the metastasis of RCC is still need to be investigated.

Sunitinib, as the first-choice tyrosine kinase inhibitor (TKI) in mRCC, provided clinical benefits for patients with mRCC [19-21]. In a phase II study of Intermittent Sunitinib in mRCC, a total of 20 patients with a median decrease in tumor burden of $45 \%$ (range 13\% to 86\%) entered the intermittent phase. Most patients exhibited a stable sawtooth pattern of tumor burden (TB) reduction while receiving Sunitinib and TB increase while not receiving Sunitinib, and metastatic progression-free survival and overall survival were 22.4 and 34.8 months, respectively [22]. Based on this finding above, Sunitinib was recognized one of the standard therapies in mRCC. However, most of patients with mRCC wouldl develop resistance to the drug after receiving the treatment eventually [23]. In order to predict treatment response, we urgently needed promising molecular biomarkers for rational indication of TKIs in patients with mRCC. Notably, our team previously reported that Sunitinib repressed RCC progression via inducing LncRNA-SARCC, which suggested that other potential non-coding RNAs were involved in RCC-Sunitinib treatment [24]. Similarly, another preclinical study reported that the efficacy of Sunitinib could be increased by miR-32-5p [25]. Then we focused on the relationship between Sunitinib and miRNAs, which the potential ability of miRNAs to predict response to TKIs in $\mathrm{mRCC}$ had not drawn enough attention.

P65, as a subunit of NF-KB, targeted a large number of genes and played an important role in the regulation of apoptosis, tumorigenesis, inflammation, and various autoimmune diseases [26]. Increased P65 activation was reported to be implicated in the development of renal cell carcinoma metastasis, promoting metastasis and progression of many cancers [27]. However, the molecular mechanism downstream of P65 had not been fully elucidated in RCC.

In our work, we demonstrated that miR-452-5p acted as a potential therapeutic target for Sunitinib and a novel tumor contributor, which promoted renal cancer progression both in vitro and in vivo. Furthermore, the transactivation of miR-452-5p in RCC was induced by P65. In addition, we explored that miR-452-5p directly bind to SMAD4 and suppress SMAD4 expression, thereby regulating SMAD4/SMAD7 signaling pathway and finally enhancing RCC cell invasion and metastasis. Here, we identified miR-452-5p as a novel tumor inducer as well as a potential diagnostic or prognostic marker in mRCC.

\section{Materials and methods}

\section{Tissue samples}

Tumor samples and paired normal tissues from RCC patients were obtained from the Department of Urology, Shanghai Tenth People's Hospital, Tongji University (Shanghai, China). The fresh tissues were kept in liquid nitrogen to protect the RNA from degradation. The current study was approved by the ethics committee of Shanghai Tenth People's Hospital.

\section{Cell culture and transfection}

The human RCC cell lines, OSRC-2 and A498 were originally purchased from Cell Bank of the Chinese Academy of Sciences (Shanghai, China). SW839 cell line was kindly provided by Dr. Chawnshang Chang from George Whipple Lab for Cancer Research, Departments of Pathology, Urology, Radiation Oncology and The Wilmot Cancer Center, University of Rochester Medical Center, Rochester, NY, 14646 USA. The SN12-PM6 cell line was kindly provided by Dr. Qingbo Huang from the Department of Urology, Chinese PLA General Hospital, Beijing, China. SW839, A498 and SN12-PM6 were cultured in Dulbecco's Modified Eagle's Medium (DMEM, Gibco, Grand Island, New York, USA) plus 10\% Fetal Bovine Serum (FBS, Hyclone, Logan, Utah, USA) with 1\% penicillin/ streptomycin (P/S, Gibco, Grand Island, New York, USA). OSRC-2 cells were cultured in RMPI 1640 (Gibco, Grand Island, New York, USA) plus 10\% fetal bovine serum (FBS, Hyclone, Logan, Utah, USA) with $1 \%$ penicillin/streptomycin (P/S, Gibco, Grand Island, New York, USA). HK-2 cells were cultured in keratinocyte medium (KM, ScienCell, San Diego, California, USA) plus $1 \%$ keratinocyte growth supplement (KGS, Scien-Cell, San Diego, California, USA) with $1 \%$ penicillin/streptomycin (P/S, ScienCell, San Diego, California, USA). All cells described above were cultured at $37{ }^{\circ} \mathrm{C}$ in the humidified $5 \% \quad \mathrm{CO} 2$ environment.

\section{Quantitative real-time PCR (qRT-PCR)}

Total RNA was extracted from tissues or cells using Trizol reagent (Invitrogen). cDNAs were synthesized with PrimeScript RT reagent Kit (Takara, Kusatsu, Japan). qRT-PCR was performed with KAPA SYBR FAST qPCR Kit (Kapa Biosystems, Woburn, Massachusetts, USA) using a 7900HT Fast Real-Time PCR System (Applied Biosystems, Carlsbad, California, USA). The primers were listed in Additional file 1: Table S1. The expression levels of miRNA were normalized to endogenous small nuclear RNA U6, and the expression levels of mRNA were normalized to endogenous control GAPDH. The $2^{-\Delta \Delta C t}$ method was used to analyze the expression levels normalized to the endogenous control. 


\section{Wound-healing assay and invasion assay}

To analyze the migration, indicated cells were plated in six-well plates. Streaks across the plate were created in the monolayer with a pipette tip. Progression of migration was observed and photographed at $0 \mathrm{~h}$ and $24 \mathrm{~h}$ after wounding. The data shown were representative micrographs of wound-healing assay of the indicated cells. The invasive capability of RCC cells was determined by the transwell assay. The membrane was coated with the matrigel (200 ng/mL; BD Biosciences). Then RCC cells were harvested and seeded with serum-free DMEM into the upper chambers at $5 \times 10^{4}$ cells/well, and the bottom chambers contained DMEM with $10 \%$ FBS, and then transwells incubated for $24 \mathrm{~h}$ at $37^{\circ} \mathrm{C}$. Following incubation, the invasive cells invading into the lower surface of the membrane were fixed by $4 \%$ paraformaldehyde and stained with $1 \%$ toluidine blue. Experiments were repeated at least three times with similar results.

\section{Cell transfection and vector construction}

According to manufacturer's protocol, miR-452-5p/ miR-NC (GenePharma, Shanghai, China), anti-miR-452-5p/ anti-miR-NC (GenePharma, Shanghai, China) and LNA-miR-452-5p/LNA-miR-NC (IBS Solutions Co. Ltd., China) were transfected at a final concentration of $50 \mathrm{nM}$ in OSRC-2 and SW839 cells using Lipofectamine 2000 (Invitrogen, Carlsbad, California, USA). In the rescue experiment, OSRC-2 and SW839 cells were co-transfected with $50 \mathrm{nM}$ oe-SMAD4 (Lingke, Shanghai, China) and $50 \mathrm{nM}$ miRNA mimics (miR-452-5p or miR-NC) with Lipofectamine 2000. The cells were harvested at $48 \mathrm{~h}$ after transfection.

Lentiviral sh-miR-452-5p/sh-miR-NC was purchased from Lingke Biotechnology (Shanghai, China). To generate the stable cell lines, OSRC-2 cell line with firefly luciferase expression was transduced with the lentiviruses all above for 2 weeks. The stably knocking down cell lines were identified using qRT-PCR.

\section{Mouse model of orthotopic tumor implantation}

OSRC-2 cells transfected with miR-452-5p, miR-452-5p + Sunitinib, sh-miR-452-5p, sh-miR-control, oe-SMAD4 and miR-452-5p + oe-SMAD4, were injected at $2 \times 10^{6}$ cells/mouse from ShanghaiSipper-BK laboratory animal Company (Shanghai, China), into the left subrenal capsule of 6-week-old male athymic nude mice. Cells were also transduced with luciferase in vivo imaging system that was performed once a week. Studies on animals were conducted with approval from the ethics committee of Shanghai Tenth People's Hospital.

\section{Chromatin immunoprecipitation assay (ChIP)}

Cells were crosslinked with $4 \%$ formaldehyde for $10 \mathrm{~min}$ followed by cell collection and sonication with a predetermined power to yield genomic DNA fragments of $300 \mathrm{bp}$ long. Lysates were precleared sequentially with normal rabbit IgG and protein A agarose. Anti-P65 antibody $(2.0 \mu \mathrm{g})$ was added to the cell lysates and incubated at $4{ }^{\circ} \mathrm{C}$ overnight. For the negative control, IgG was used in the reaction. Specific primer sets designed to amplify a target sequence within human miR-452-5p promoter were listed in Additional file 1: Table S1; PCR products were analyzed by agarose gel electrophoresis.

\section{Luciferase reporter assay}

To confirm whether P65 could increase miR-452-5p promoter activity, OSRC-2 cells transfected with oe-P65 or vector cultured in 48-well plates were co-transfected with $1.5 \mu \mathrm{g}$ of firefly luciferase reporter and $0.35 \mathrm{ng}$ Renilla luciferase reporter with Lipofectamine 2000. Luciferase reporter assay used the one step directed cloning kit (Novoprotein, Shanghai, China) according to the manufacturer's manual. To identify potential downstream genes of miR-452-5p, luciferase reporter assay was performed as the above described. In briefly, OSRC-2 cells transfected with miR-452-5p or vector cultured in 48-well plates were cotransfected with $1.5 \mu \mathrm{g}$ of firefly luciferase reporter and $0.35 \mathrm{ng}$ Renilla luciferase reporter with Lipofectamine 2000 regents. Luciferase reporter assay using the one step directed cloning kit.

\section{Protein extracting and Western blotting analysis}

The cells were lysed using RIPA buffer plus protease inhibitors and phosphatase inhibitors. For western blot analysis, $25 \mu \mathrm{g}$ of protein extracts were loaded to $10 \%$ sodium dodecylsulfate-polyacrylamide gel electrophoresis gels and transferred to nitrocellulose membranes. The membranes were incubated with a primary antibody overnight and were incubated with a secondary antibody in $1 \mathrm{~h}$ with room temperature. The expression of $\beta$-actin was used as loading control. The information of antibodies were listed as follow: SMAD4(1:1000, Sangon Biotech, China, D220124), SMAD7 (1:1000, Sangon Biotech, China, D160746).

\section{Immunohistochemistry}

Immunohistochemistry was performed using a primary antibody against SMAD4 (1:800, Abcam, ab129108), SMAD7 (1:100, Abcam, ab131443), The degree of positivity was initially classified according to scoring both the proportion of positive staining tumor cells and the staining intensities. Scores representing the proportion of positively stained tumor cells were graded as: $0(<10 \%) ; 1$ (11-25\%); 2 (26-50\%); 3 (51-75\%) and 4 (>75\%). The intensity of staining was determined as: 0 (no staining); 1 (weak staining $=$ light yellow); 2 (moderate staining = yellow brown); and 3 (strong staining = brown). The staining index was calculated as the product of staining 
intensity $\times$ percentage of positive tumor cells, resulting in scores of $0,1,2,3,4,6,8,9$ and 12 . Only cells with clear tumor cell morphology were scored.

\section{Statistical analysis}

Results are expressed at least 3 independent experiments. Using the GraphPad Prism statistical program, data were analyzed using ANOVA or Student' t test unless otherwise specified. $P$ values $<0.05$ were considered significant.

\section{Result}

\section{Sunitinib abrogates RCC cell invasion and metastasis via depressing miR-452-5p}

Our team had previously reported that Sunitinib remarkably blunted RCC progression via inducing LncRNA-SARCC [24]. In an attempt to further explore whether Sunitinib inhibited RCC cell invasion and metastasis in a miRNA-dependent manner, we first focused on 2 microarray data, GSE32099 (differentially expressed miRNAs in peripheral blood under Sunitinib treatment, Additional file 2: Table S2) and GSE37989 (metastasis-associated miRNAs, Additional file 3: Table S3) through searching GEO datasets (Fig. 1a). Next we selected out top 10 common miRNAs, which were downregulated after Sunitinib treatment while upregulated in metastasis tissues (Fig. 1b). Notably, two potential candidate miRNAs (miRNA-452-5p and miRNA-605-5p) were selected on the basis of their involvement in RCC tumorigenesis by using OncomiR, an online resource for exploring miRNA dysregulation in cancer. As shown in Fig. 1c, we used qRT-PCR to detect both miRNAs expression under Sunitinib treatment $(5 \mu \mathrm{M}$ and $10 \mu \mathrm{M})$ and finally selected out miRNA-452-5p as a validation target in OSRC-2 and SW839 cell lines.

To confirm whether Sunitinib modulated miR-452-5p to impact its therapeutic effect, we first established RCC cell lines with miR-452-5p mimic, including OSRC-2 and SW839. Transwell invasion assay suggested that miR-452-5p mimic-transduced both cell lines contributed to more invasiveness compared with cells with miR-NC group. Consistently, wound healing assay also indicated that miR-452-5p overexpression saliently enhanced cell migration than miR-NC group. Next, we prepared both cells lines with miR-452-5p mimic and miR-NC and then treated them with $10 \mu \mathrm{M}$ Sunitinib for $24 \mathrm{~h}$. As shown in Fig. 1d and e, cells with miR-452-5p mimic performed more migratory capability compared with cells with control using wound-healing assay. Similarly, cells transfected with miR-452-5p mimic owned more invasive ability than those with control group (Fig. If and g). Both pro-metastatic phenotype above elicited that miR-452-5p was potential one of the key mediators to influence Sunitinib efficacy.
To further confirm that miR-452-5p might act as a metastatic-promoting miRNA, we then established miR-452-5p with Sunitinib in a xenograft model. OSRC-2 cells with firefly luciferase expression were transfected with miR-NC or miR-452-5p under Sunitinib $10 \mu \mathrm{M}$ treatment. The stable clones were injected into left renal capsule of nude mice and metastatic sites were measured. As shown in the Fig. 1h-i, Sunitinib blunted metastatic sites, which was partially reversed by miR-452-5p.

Taken together, the results above suggested that miR-452-5p might be involved in Sunitinib repressing RCC invasion and metastasis.

\section{miR-452-5p is associated with poor prognosis of RCC}

To further understand the expression and clinical relevance of miR-452-5p in RCC, the tumor development and survival outcome data were collected from The Cancer Genome Atlas (TCGA) database (http://cancerg enome.nih.gov/). The results illustrated that the expression of miR-452-5p was pronouncedly increased in RCC tumor tissue compared to normal tissues $(p<0.001)$ (Fig. $1 \mathrm{j}$ and Additional file 4: Table S4). In addition, qRT-PCR was performed in renal tumors and paired non-cancerous tissues from $20 \mathrm{RCC}$ patients. The results demonstrated that the expression of miR-452-5p was substantially increased in RCC compared to adjacent non-cancerous tissues $(p<0.05)$ (Fig. $1 \mathrm{k}$ and 1$)$. As expected, the expression of miR-452-5p was remarkably expressed in various RCC cell lines, involving SW839, A498, SN12-PM6 and OSRC-2 compared to normal adult human kidney HK-2 cells $(p<0.01)$ (Fig. $1 \mathrm{~m})$. Furthermore, receiver operating characteristics (ROC) analysis revealed that miR-452-5p might serve as a useful biomarker for discriminating RCC from normal tissues with an area under the ROC curve (AUC) of 0.919 (95\% CI, 0.708-0.993; Fig. 1n). Next, we analyzed the association between miR-452-5p expression and the clinicopathological characteristics from 102 RCC patients (Additional file 5: Table S5). Correlation regression analysis showed that high expression of miR-452-5p was significantly correlated with metastasis $(p=0.043)$. We further performed univariate and multivariable logistic regression models to analyze the correlation of miR-452-5p levels with overall survival of RCC patients. Patient characteristics were provided in Additional file 6: Table S6. Univariate analysis proved that a higher miR-452-5p expression (hazard ratio, $\mathrm{HR}=1.56$; $95 \%$ confidence interval, $\mathrm{CI}=1.08-2.25 ; p=0.018)$ and metastasis $(\mathrm{HR}=1.64 ; 95 \% \mathrm{CI}=1.25-1.86 ; p=0.036)$ were clearly correlated with overall survival. Multivariate analysis indicated that a higher miR-452-5p expression $(\mathrm{HR}=1.58 ; 95 \% \mathrm{CI}=1.07-2.31 ; p=0.020)$ were markedly associated with overall survival. In addition, 


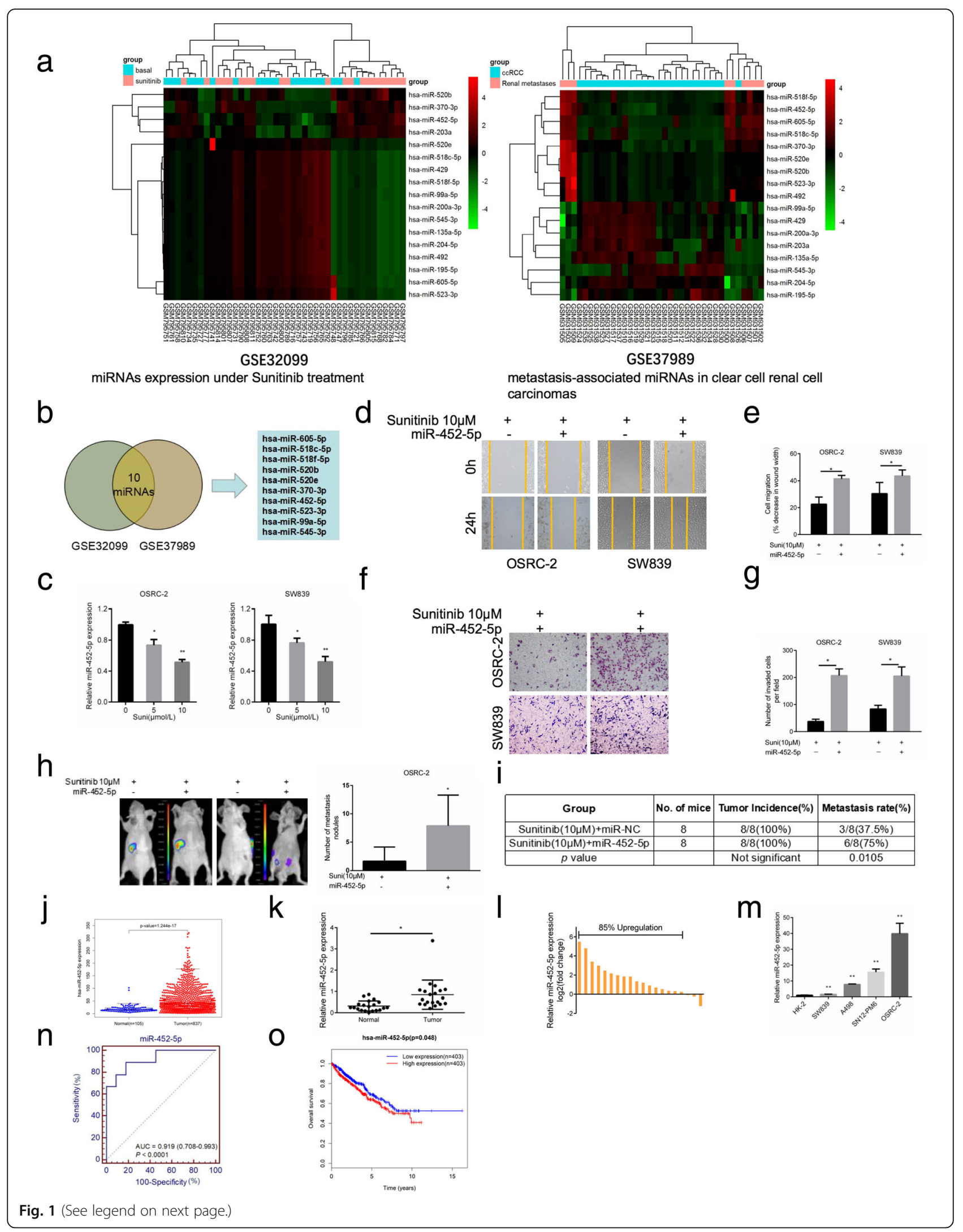


(See figure on previous page.)

Fig. 1 Sunitinib abrogates RCC cell invasion and metastasis via depressing miR-452-5p. a Presented are heatmap of the most differentially expressed miRNAs in peripheral blood under Sunitinib treatment (GSE32099) and between tumor tissue and pair-matched normal tissues(GSE37989). b The above TCGA analysis showed 10 miRNAs were significantly differentially expressed both in GSE32099 and GSE37989. c qRT-PCR assays for miR-452-5p expression with 0,5 and $10 \mu \mathrm{M}$ Sunitinib treatment in OSRC-2 and SW839 cells. d-e Representative micrographs of wound-healing assay and decrease in wound width was induced by transfection of miR-452-5p in OSRC-2 and SW839 cells versus miR-NC cells with or without treatment of $10 \mu \mathrm{M}$ Sunitinib. $\mathbf{f}-\mathbf{g}$ Representative images and number of invasive cells per high-power field was induced by transfection of miR-452-5p in OSRC-2 and SW839 cells versus miR-NC cells with or without treatment of $10 \mu \mathrm{M}$ Sunitinib. $\mathbf{h}$ Orthotopic xenograft animal models were generated using miR-452-5p or miR-NC in OSRC-2 cells and mice treated with $10 \mu \mathrm{M}$ Sunitinib. Presented are representative images (left) of abdominal metastasis viewed by IVIS system in each group 4 weeks after the orthotopic xenograft transplantation ( $n=8)$ and Quantitation of metastasis nodules shown at right. $\mathbf{i}$ Incidence of metastasis in orthotopic xenografts after 4 weeks. $\mathbf{j}$ TCGA cohort analysis of the differentially expressed levels of miR-452-5p in RCC tumor samples and pair-matched normal tissues. Each dot represents one sample. $\mathbf{k}$ Comparison of miR-452-5p expression in 20 paired RCC tumor tissues and adjacent normal tissues via qRT-PCR. I Relative miR-452-5p expression levels in RCC samples are presented as fold change $=2$ ( $\Delta C \mathrm{Ct}$ normal- $\Delta \mathrm{Ct}$ tumor) of tumor versus matched normal tissues, $85 \%$ of which was upregulated in tumor tissues than adjacent normal tissues. $\mathbf{m}$ miR-452-5p expression in a series of RCC cell lines (SW839, A498, SN12-PM6 and OSRC-2) and human normal renal tubular epithelial cell line HK-2. $\mathbf{n}$ ROC analysis to assess the specificity and sensitivity of miR-452-5p to differentiate between RCC and normal tissues. o Kaplan-Meier analyses of the correlations between miR-452-5p expression level and overall survival of 806 patients with RCC through TCGA analysis. The median expression level was used as the cutoff. Data shown are mean \pm S.D. ${ }^{*} p<0.05,{ }^{*} p<0.01$

Kaplan-Meier survival analysis indicated that RCC patients with the lower levels of miR-452-5p had longer overall survival than those with the higher levels of miR-452-5p $(p<0.05)$ (Fig. 10 and Additional file 7: Table S7).

In conclusion, the above data indicated that higher level of miR-452-5p was obviously correlated with poor prognosis of RCC.

\section{miR-452-5p-inhibition silences the development of pro- metastatic phenotype in vitro}

Previously we proved that miR-452-5p facilitated cell migration and invasion in OSRC-2 and SW839 cells. To identify and characterize the biological function of miR-452-5p in RCC development, anti-miR-452-5p or anti-miR-NC were transfected into both RCC cells to investigate the anti-migratory and anti-invasive role of silenced miR-452-5p in renal cancer (Fig. 2a). Compared to control group, anti-miR-452-5p could effectively inhibit migration and invasion in both RCC cells (Fig. 2b-e). Furthermore, locked nucleic acid technology (LNA) was used to both cell lines to interrupt the expression of miR-452-5p (Fig. 2f). The invasion and migration assay suggested the same tendency, which coincided with previous outcomes (Fig. 2g-j).

Taken together, the above data identified that miR-452-5p functioned as a metastasis-promoting miRNA in RCC.

\section{miR-452-5p level is maximized by P65 through directly binding its promoter}

To investigate the mechanism responsible for the up-regulation of miR-452-5p in RCC, we predicted 4 potential transcription factors, involving AR, P65 (RELA), HOXD9 and POU2F2, in the promoter region of miR-452-5p using PROMO, and using two miRNA target-predicting algorithms including Target Scan and miTar to narrow the candidates (Fig. 3a). Then, UAL$\mathrm{CAN}$, an interactive web resource for analyzing cancer transcriptome data, was used to assess the above 4 transcription factors. Especially, AR and HOXD9 were ruled out due to their lower expression in RCC tissues than in normal renal tissues.

Next, we applied PROMO to determine the two transcription factors (P65 and POU2F2) binding sites of miR-452-5p within 3000 bases upstream from the transcriptional start site (Fig. 3b). ChIP assay elucidated that P65 could bind to the promoter of miR-452-5p, while POU2F2 failed to bind (Fig. 3c). Furthermore, luciferase assay confirmed that P65 increased miR-452-5p promoter activity, yet, this tendency abolished in miR-452-5p promoter-mutant OSRC-2 cells (Fig. 3d and e).

Interestingly, data from TCGA database confirmed positive correlation between $\mathrm{P} 65$ and miR-452-5p expression $(p<0.001)$ (Fig. 3f and Additional file 8: Table S8). In parallel, P65 expression was high expressed in RCC tumor tissues than in normal tissues $(p<0.001)$ (Fig. 3g and Additional file 9: Table S9). In addition, Immunohistochemical (IHC) staining of P65 protein level indicated a higher expression in RCC tissues than in adjacent normal tissues (Fig. 3h). Notably, TCGA database also showed higher P65 levels were obviously correlated with worse TNM stage (Fig. 3i and Additional file 10: Table S10). Kaplan-Meier survival analysis certified that patients with higher P65 levels had poor overall survival time than those with lower P65 levels from TCGA database $(p<0.05)$ (Fig. 3j and Additional file 11: Table S11).

In brief, these results above corroborated that P65 were a carcinogenic gene and might induced miR-452-5p transcriptional level via directly binding its promoter in RCC. 


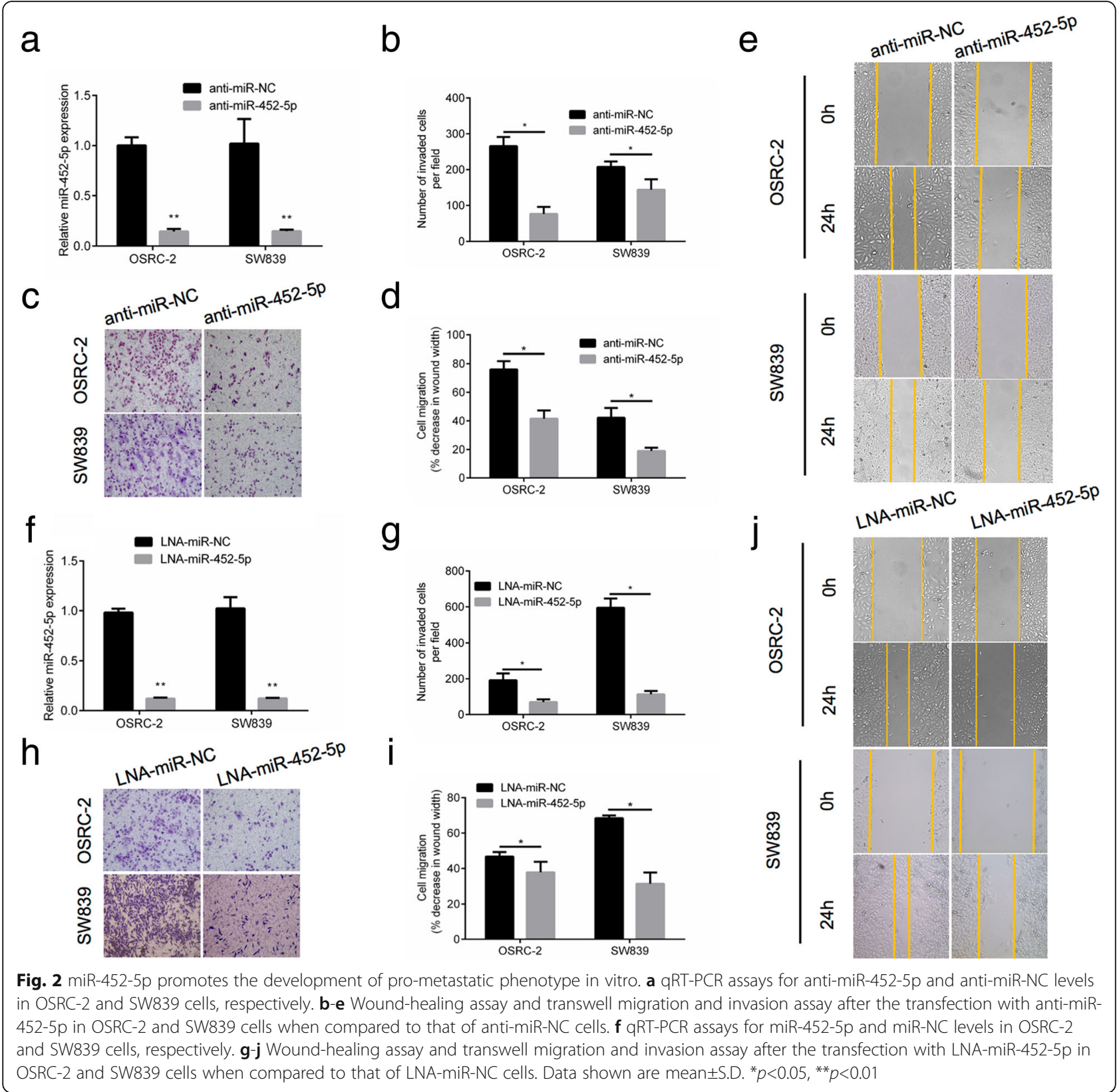

SMAD4 is the target gene of miR-452-5p and associated with good prognosis of RCC

To further dissect the mechanism underlying miR-452-5p modulating induction of RCC metastasis, we searched for potential downstream genes of miR-452-5p through four different miRNA target-predicting algorithms including TargetMiner, miRTarBase, miRWalk and miRTar, then focused on the one possible candidate target gene SMAD4 (Fig. 4a). TCGA data sets suggested SMAD4 was low expressed in RCC tissues compared with normal tissues $(p<0.05)$ (Fig. 4b and Additional file 12: Table S12). In particular, IHC staining of SMAD4 expression in RCC tissues and normal tissues also elucidated the same tendency with the above results (Fig. 4c). Additionally, KaplanMeier survival analysis proved that patients with lower SMAD4 levels had poor overall survival time than those with higher SMAD4 levels from TCGA database $(p<0.05)$ (Fig. 4d and Additional file 13: Table S13). Data from TCGA database revealed that there is a negative correlation between miR-452-5p and SMAD4, in keeping with the notion that miRNAs negatively regulate gene expression (Fig. 4e and Additional file 14: Table S14). In order to verify the speculation, by a computational prediction of miRNA databases, we identified three putative binding sites of miR-452-5p with high complementarity in SMAD4 promoter region (Fig. 4f). To identify whether 

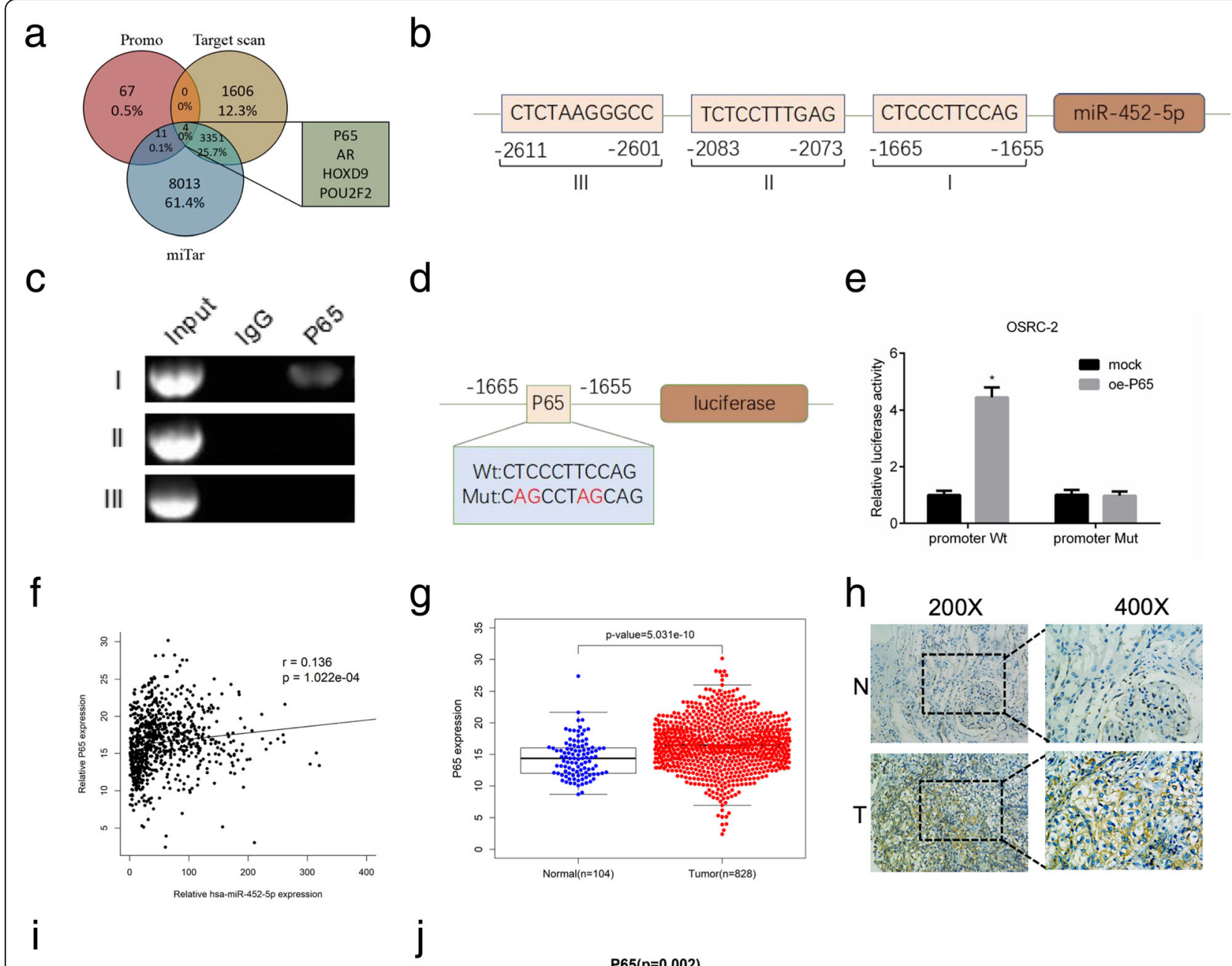

d

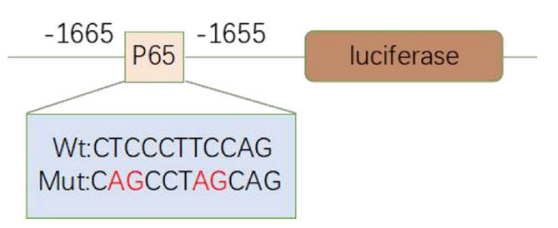

g

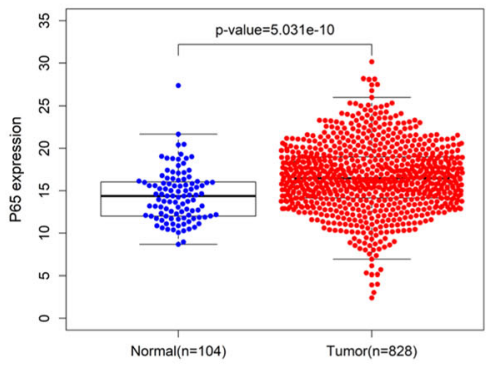

j

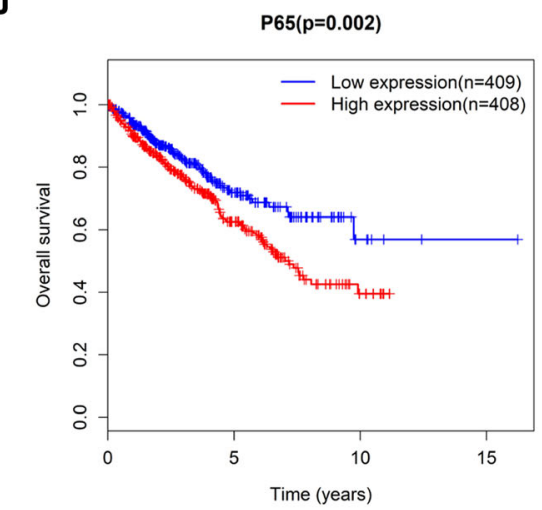

e

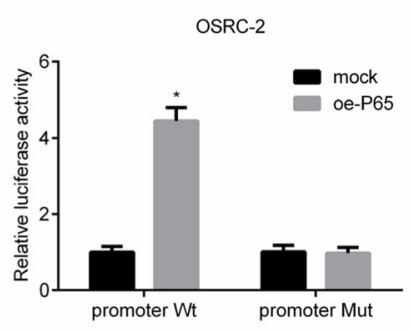

h

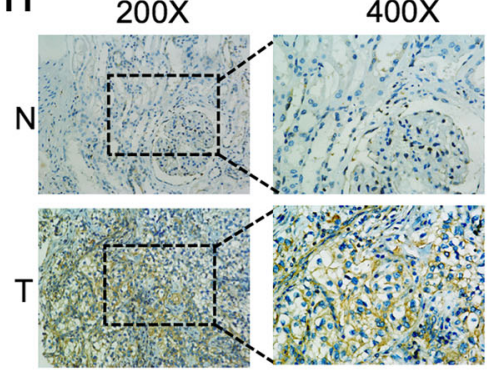

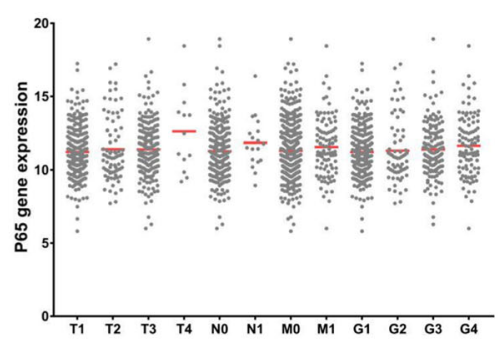

Fig. 3 miR-452-5p level is maximized by P65 through directly binding its promoter. a The schematic illustration of the proposed model depicting of 4 putative upstream transcription factors of miR-452-5p from 3 prediction databases, Promo, TargetScan and miTar. b Bioinformatic analysis of potential P65 binding sites in miR-452-5p promoter. $\mathbf{c}$ Lysates of OSRC-2 cells were subjected to ChIP assay. $\mathbf{d}$ The schematic illustration of P65$\mathrm{wt} /$ mut in the miR-452-5p promoter. e Luciferase reporter assays in OSRC-2 cells. $\mathbf{f}$ the correlation between miR-452-5p expression and corresponding P65 expression in RCC and normal renal tissues from TCGA RCC dataset. $\mathbf{g}$ P65 expression in RCC and normal renal tissues from TCGA RCC dataset. $\mathbf{h} 1 \mathrm{HC}$ staining revealed the level of P65 expression in RCC tissues $(\times 200, \times 400)$ versus adjacent normal tissues. i P65 expression in different TNM stage of RCC samples from TCGA RCC dataset. $\mathbf{j}$ P65 expression and patients' survival time from TCGA RCC dataset. Data shown are mean \pm S.D. ${ }^{*} p<0.05$ 


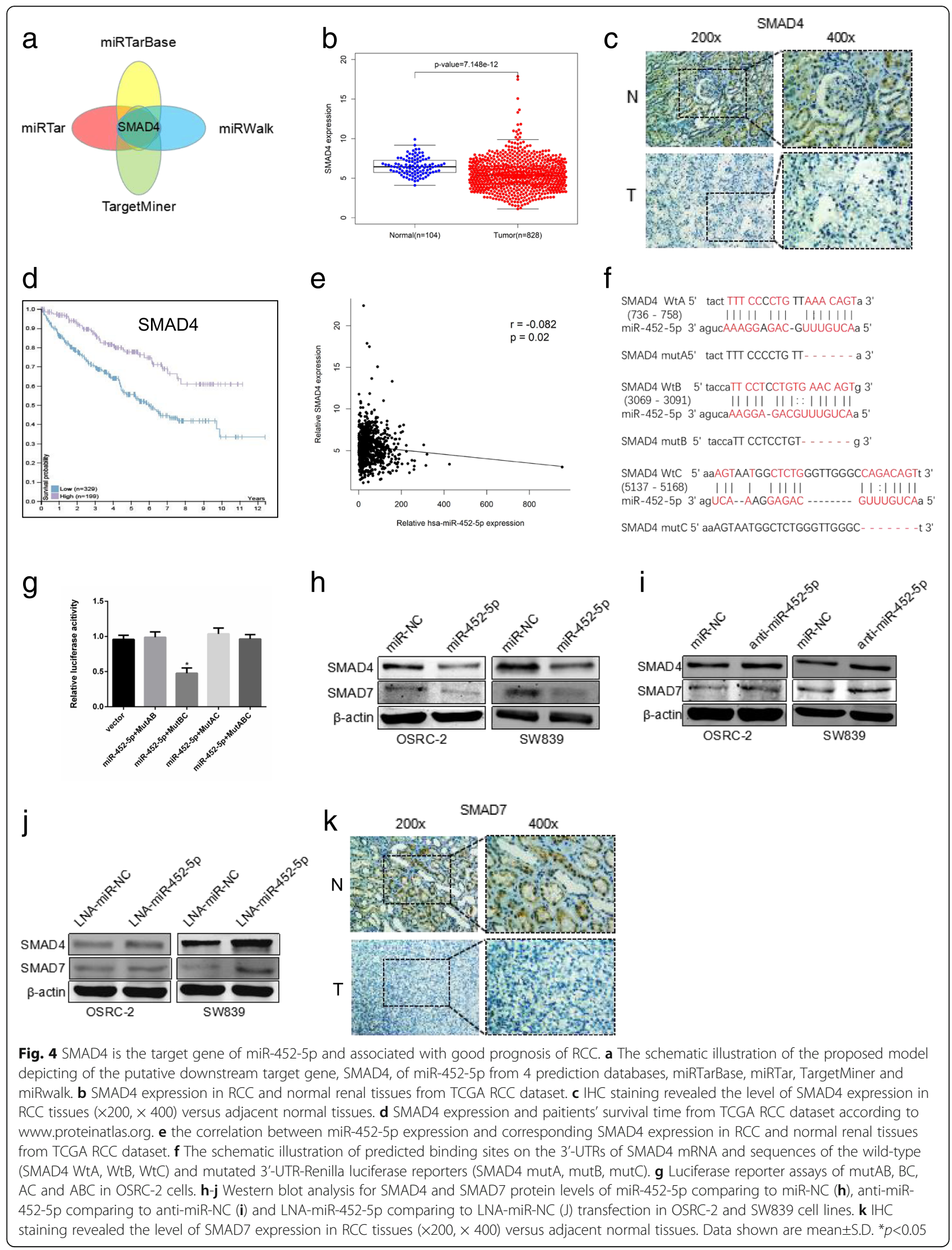




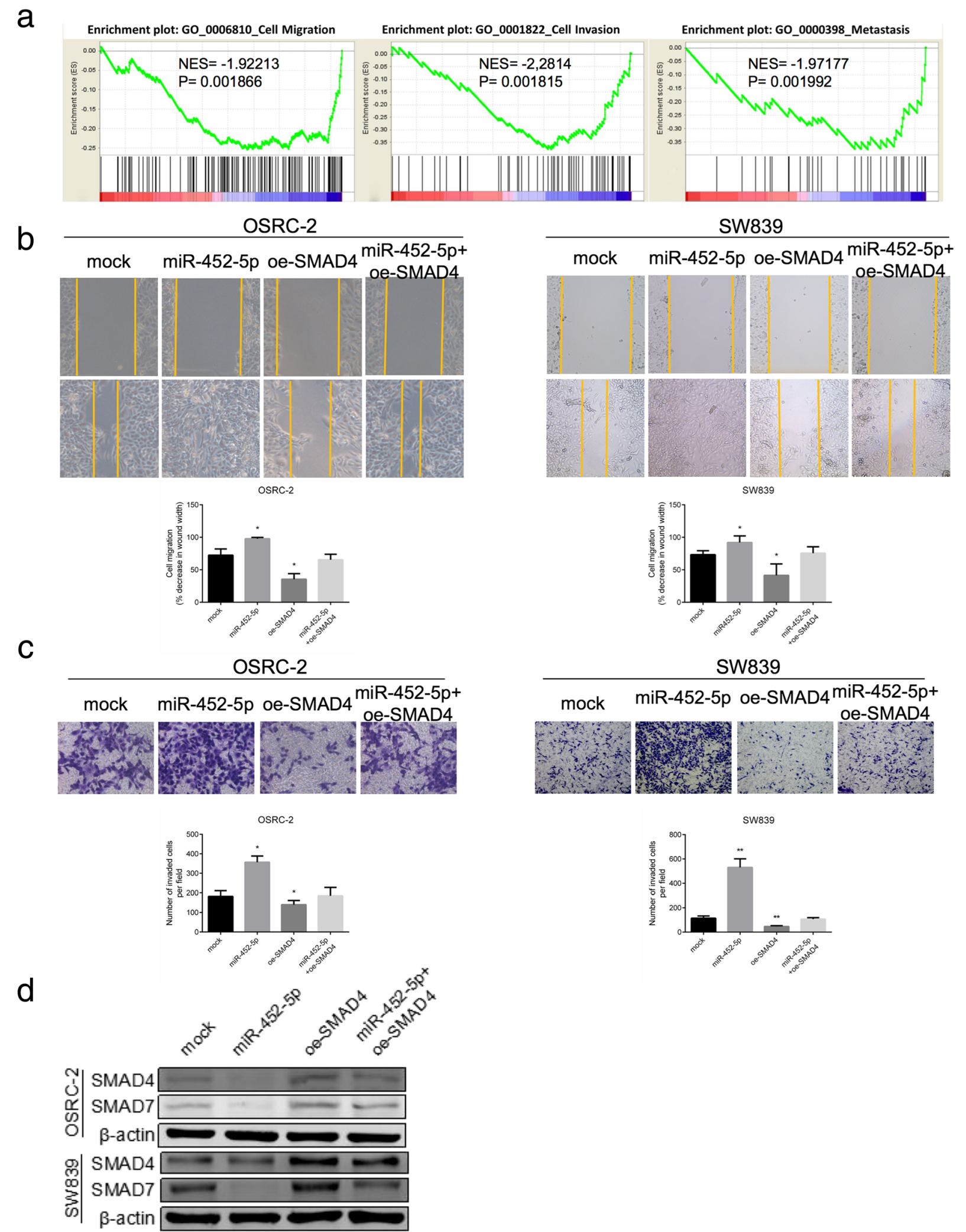

Fig. 5 (See legend on next page.) 
(See figure on previous page.)

Fig. 5 SMAD4 recapitulates the effects of miR-452-5p in RCC cells. a GSEA of published gene array, GO:0006810, GO:0001822 and GO:0000398 dataset referred to migration, invasion and metastasis related-gene signatures, respectively. NES, normalized enrichment score. $\mathbf{b}$ Cell migration in OSRC-2 and SW839 cells were analyzed by wound-healing assay. Cells were respectively transfected with vector, miR-452-5p, oe-SMAD4 and miR452-5p+oe-SMAD4. c Cell invasion in OSRC-2 and SW839 cells were analyzed by transwell assays. Cells were respectively transfected with vector, miR-452-5p, oe-SMAD4 and miR-452-5p+oe-SMAD4. $\mathbf{d}$ The protein levels of SMAD4 and SMAD7 in OSRC-2 and SW839 cells were assessed by Western blot analysis. Respectively, cells were transfected with vector, miR-452-5p, oe-SMAD4 and miR-452-5p+oe-SMAD4 for 48 hours before detection. Data shown are mean \pm S.D. ${ }^{*} p<0.05,{ }^{* *} p<0.01$

Wt-A, Wt-B or Wt-C was functional, luciferase reporter assays was performed and results demonstrated that only A binding site on the 3'-UTR of SMAD4 mRNA was functional. (Fig. 4g). As a former study described, SMAD7 might be a feasible downstream gene of SMAD4 [28]. Consequently, WB analysis was used to detect the SMAD4 and SMAD7 protein level. When we introduced miR-452-5p mimic into OSRC-2 and SW839 cell lines, the decrease in SMAD4 and SMAD7 protein was confirmed (Fig. 4h). Conversely, both inhibited miR-452-5p and LNA-miR-452-5p markedly enhanced their protein level (Fig. 4i and j). Histological analysis of SMAD7 protein status in RCC tissues and normal tissues also elucidated that SMAD7 was potently downregulated in RCC tissues but was nearly undetectable in normal renal tissues (Fig. 4k).

Together, the data above revealed that miR-452-5p directly targeted SMAD4 and minimized SMAD4 and SMAD7 expression in RCC cells.

\section{SMAD4 recapitulates the effects of miR-452-5p in RCC cells}

To examine whether SMAD4 could suppress cell invasion and metastasis in RCC, we performed gene set enrichment analysis with published gene array of metastatic RCC signatures (GSE12606), and results revealed that SMAD4 expression was negatively correlated with RCC cell migration, invasion and metastasis (Fig. 5a). As previously described, we observed that miR-452-5p remarkably increased the cell migration and invasion of OSRC-2 and SW839 cells compared with mock. Importantly, an interruption approach with oe-SMAD4 partially reversed the effects of miR-452-5p on cell migration and invasion (Fig. 5b and c). As we expected, WB confirmed that oe-SMAD4 recapitulated the effect of blocked SMAD4 and SMAD7 protein caused by miR-452-5p in both cell lines (Fig. 5d).

Together, these results above suggested that miR-452-5p promoted RCC cell invasion and metastasis through suppressing SMAD4.

\section{miR-452-5p promotes RCC metastasis through targeting SMAD4 in vivo}

To further validate that miR-452-5p might act as a tumor inducer in vivo, we inoculated different clones of OSRC- 2 cells. In this model system, sh-miR-452-5p cells as well as its control cells were inoculated into the left kidney capsule of xenograft. As shown in Fig. 6a, a dramatic induction of luciferase expression in tumors of both groups was detected by in vivo imaging system (IVIS) as early as the 2 th week. Figure $6 \mathrm{~b}$ and $\mathrm{c}$ showed promotion of tumor metastases in the sh-miR-NC group compared with the sh-miR-452-5p group after 4 weeks (Fig. 6b and c). Furthermore, sh-miR-452-5p attenuated lung, liver, spleen and right renal metastases (Fig. 6d). In parallel, IHC staining substantiated SMAD4 and SMAD7 protein levels of sh-miR-452-5p group increased compared with sh-miR-NC group in renal tumor tissues from nude mice (Fig. 6e). Conversely, transfection of miR-452-5p into OSRC-2 cells led to sufficiently enhanced metastasis of orthotopic xenograft tumors, whereas oe-SMAD4 into miR-452-5p upregulated cells mostly abolished this induction (Fig. 6f-h).

Together, results from Fig. 6 illustrated that SMAD4-dependent miR-452-5p functioned as a critical tumor-metastasis promoter in RCC.

\section{Discussion}

In our study, we first reported that miR-452-5p, induced by $\mathrm{P} 65$, was a potential therapeutic target for Sunitinib and associated with mRCC poor prognosis. MiR-452-5p in RCC samples and cell lines was high expressed compared with that in surrounding non-tumor tissues as well as HK-2 normal cells. Moreover, P65 could directly bind to the promoter of miR-452-5p and transcriptionally induce miR-452-5p expression. Consistently, miR-452-5p elevated cell migration and invasion in RCC cell lines and promoted RCC progression through targeting SMAD4/SMAD7 signals. All these results supported the conclusion that miR-452-5p acted as a tumor inducer and a metastasis-promoting miRNA in mRCC.

Previously, some reports demonstrated that miR-452-5p might function as a tumorigenesis-promoting in hepatocellular carcinoma and contribute to the docetaxel resistance of breast cancer cells $[15,17]$. On the other hand, it had also been reported that expression of miR-452-5p inhibited metastasis in osteosarcoma, non-small cell lung cancer, and down-regulation of miR-452-5p was associated with adriamycin-resistance in breast cancer cells $[14,16,29]$. The possible reason for the opposite role of miR-452-5p might be related to various human cancers 


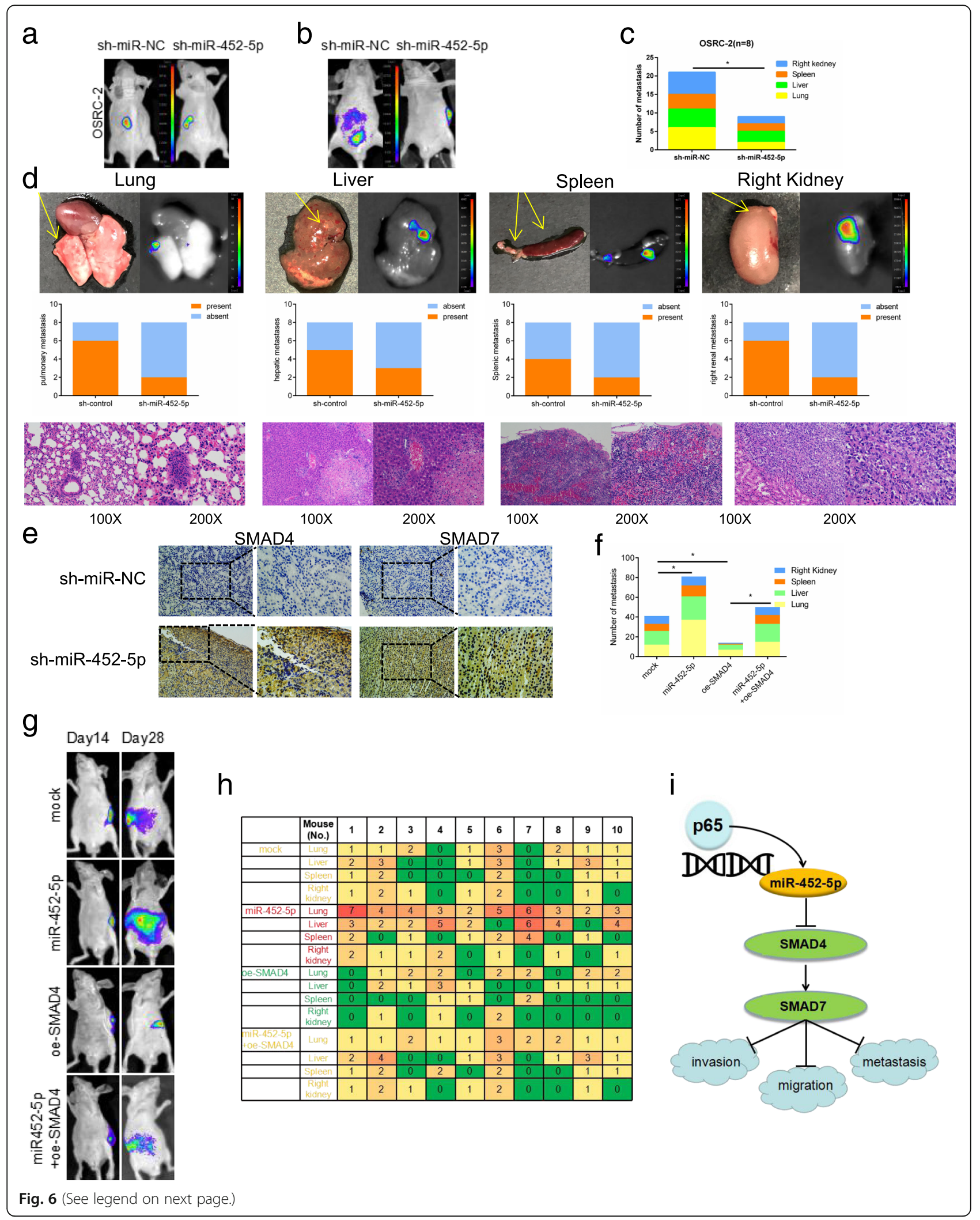




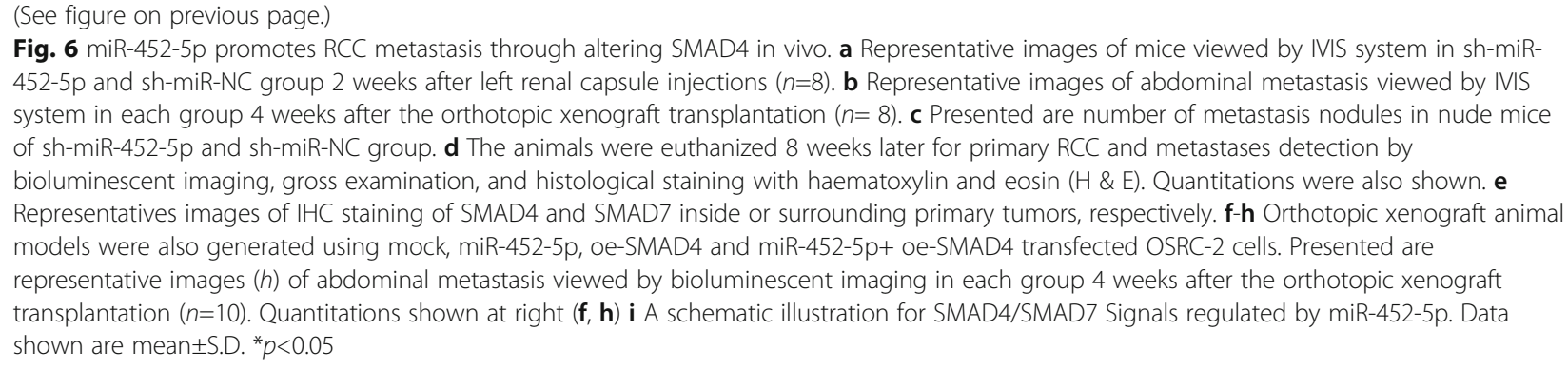

or drug treatment. Importantly, the relationship between $\mathrm{mRCC}$ and miR-452-5p as well as its response to Sunitinib remained unknown. In this work, our studies reported that Sunitinib attenuated miR-452-5p to impact its therapeutic effect, and miR-452-5p enhanced the development of pro-metastatic phenotype in mRCC.

According to early reports, P65, the central component of NF-KB pathway, had been suggested to act as a transcription factor in various kinds of diseases [30-33]. Dong Yang et al. identified that P65 induced miR-17 transcription by binding its promoter elements to regulate proliferation of vascular smooth muscle cells under inflammation [34]. Rezapour S et al. reported that P65 was constitutively activated in colorectal cancer [30]. Other reports proved that P65 might serve as an activating transcription factor in several types of human cancers $[35,36]$. Our conclusions supported these findings above that P65 could directly bind to the miR-452-5p promoter and thus transcriptionally induce miR-452-5p expression. Conversely, it was also reported that P65 could attenuate transcriptional activity under a certain condition. Raman P. NAGARAJAN et al. validated that P65 was able to inhibit the SMAD7 promoter activity [28], which, in some extent, was also coincided with our result that P65 induced miR-452-5p transcription, which targeted SMAD4 and repressed SMAD7 expression. Our study further illustrated that high P65 expression was obviously correlated with higher clinical TMN stage and contributed to poor prognosis in RCC patients. All these results concluded that P65 served as a tumor-inducer in RCC.

As a member of SMAD family, SMAD4 was a critical component of TGF- $\beta$ signaling and got involved in MAPK, CDK and PI3K signaling [37, 38]. Although it was believed that a complex containing SMAD4 formed by TGF- $\beta$ stimulation was translocated into the nucleus, then EMT was induced [39]. However, data from TCGA database showed that SMAD4 was downregulated in RCC tissues compared with normal tissues and patients with lower SMAD4 levels had worse overall survival time. As we all know, SMAD4 had been recognized as a tumor suppressor gene in a good deal of cancers, and recent study had presented that SMAD4 suppressed the progression of RCC by targeting various downstream genes [40-42]. The reason for such inconsistency remained an enigma. Here, we elucidated that miR-452-5p directly targeted SMAD4, and as a downstream gene, SMAD7 was also repressed. Furthermore, disruption of SMAD7 had been reported to play a pivotal role in EMT, which finally contributed to tumor metastasis [43-45]. Our study presented a road map that miR-452-5p facilitated RCC invasion and metastasis through SMAD4/SMAD7 Signals.

Although Sunitinib was the first-line treatment for mRCC, the clinical benefit of Sunitinib was limited, and the vast majority of mRCC patients under sunitinib treatment ultimately developed disease progression because of the acquisition of resistance. Several studies reported that miRNAs played a crucial role in alterting the sensitivity to Sunitinib in multiple tumors, indicating that miRNAs were potential therapeutic targets for Sunitinib,and miRNA modulation combined with Sunitinib as a novel therapeutic strategy was under exploring [46-49]. Notably, the mechanistic pathway of how the tyrosine kinase inhibitor could down regulate miRNA-452-5p remained unclear. It's a pity that we did not conduct further study on this mechanism. However, a previous study from our team confirmed that Sunitinib repressed RCC progression via inducing LncRNA-SARCC, which gave us a hint that some LncRNAs might get involved in the progress [24]. On the other hand, some transcription factors like HIF1 $\alpha$ might be responsible to Sunitinib treatment as well [50]. From the above, the tyrosine kinase inhibitor might downregulated miRNA-452-5p through non-coding RNAs or transcription factors. In our study, miR-452-5p was confirmed to determine the sensitivity to Sunitinib, and high expression of miR-452-5p was suggested to contribute to Sunitinib resistance. Therefore, it could be used to divide mRCC patients into different responsive groups to save both money and time for the non-responsive patients and enhance Sunitinib treatment efficacy through down-regulating the expression of miR-452-5p.

In conclusion, we conclude that Sunitinib efficacy is potentially connected with miR-452-5p, which acts as an efficient metastasis-promoter through SMAD4/SMAD7 signals in RCC patients. This finding points to a novel therapeutic target to maximize Sunitinib efficiency in 
RCC, and helps us to better suppress RCC progression via targeting this newly identified signal pathway.

\section{Additional files}

Additional file 1: Table S1. Sequences of oligonucleotides used in this study. (XLSX $10 \mathrm{~kb}$ )

Additional file 2: Table S2-GSE32099. (XLS $113 \mathrm{~kb}$ )

Additional file 3: Table S3-GSE37989. (XLS 72 kb)

Additional file 4: Table S4. miR-452-5p expression in RCC and normal renal samples from TCGA RCC dataset. (XLS $137 \mathrm{~kb}$ )

Additional file 5: Table S5. Clinical characteristics of 102 RCC patients according to miRNA-452-5p expression levels. (XLSX $12 \mathrm{~kb}$ )

Additional file 6: Table S6. Univariate and Multivariate Cox Regression Analyses of miR-452-5p. (XLSX $10 \mathrm{~kb})$

Additional file 7: Table S7. miR-532-5p expression and paitients' survival time from TCGA RCC dataset. (XLS $114 \mathrm{~kb}$ )

Additional file 8: Table S8. miR-452-5p expression and corresponding P65 expression in RCC and normal renal samples from TCGA RCC dataset. (XLSX $38.1 \mathrm{~kb}$ )

Additional file 9: Table S9. P65 expression in RCC and normal renal samples from TCGA RCC dataset. (XLS $136 \mathrm{~kb}$ )

Additional file 10: Table S10. P65 expression in different TNM stage of RCC samples from TCGA RCC dataset. (XLSX $40 \mathrm{~kb}$ )

Additional file 11: Table S11. P65 expression and patients' survival time from TCGA RCC dataset. (XLSX $42 \mathrm{~kb}$ )

Additional file 12: Table S12. SMAD4 expression in RCC and normal renal samples from TCGA RCC dataset. (XLSX $40 \mathrm{~kb}$ )

Additional file 13: Table S13. SMAD4 expression and paitients' survival time from TCGA RCC dataset according to www.proteinatlas.org. (XLSX $31 \mathrm{~kb}$ )

Additional file 14: Table S14. miR-452-5p expression and corresponding SMAD4 expression in RCC and normal renal samples from TCGA RCC dataset. (XLSX $42 \mathrm{~kb}$ )

\section{Abbreviations}

AUC: Area under the ROC curve; ChIP: Chromatin immunoprecipitation assay; EMT: Epithelial-Mesenchymal Transition; IF: Immunofluorescence;

IHC: Immunohistochemical; IVIS: in vivo imaging system; LNA: Locked nucleic acid technology; miRNAs: microRNAs; mRCC: Metastasis renal cell carcinoma; qRT-PCR: Quantitative real-time PCR; RCC: Renal cell carcinoma;

ROC: Receiver operating characteristics; TB: Tumor burden; TCGA: The Cancer Genome Atlas; TKI: Targeted receptor tyrosine kinase inhibitor

\section{Acknowledgements}

We thank Genminix Informatics co., Ltd. for its bioinformatic analysis in this manuscript.

\section{Funding}

This work was sponsored by the National Natural Science Foundation of China (31570775, 81602216 and 81772705), Natural Science Foundation of Shanghai (16ZR1426500), and Shanghai Pujiang Program (16PJD037). Three Year Plan of Action Program from Shen Kang (16CR3062B), Cultivation Program of Renji Hospital (PYZY16-006).

\section{Availability of data and materials}

Data and material is available at the Molecular Cancer's website.

\section{Authors' contributions}

Conception and design: YX, WZ and WX; Experimental operation: SL, JZ, YC; Provision of materials or patients' information: JZ, JM, DG, WK; Collection and assembly of data: WZ, SL and DG; Manuscript writing: WZ and SL; Manuscript revision: WZ, JM and JZ; Final approval of manuscript: All authors.

\section{Ethics approval and consent to participate}

This study was approved by the Shanghai Tenth People's hospital ethics committee and written informed consent was obtained from all patients. All animal studies were approved by the Institutional Animal Care and Use Committee of the Shanghai Tenth People's Hospital.

\section{Consent for publication}

Not applicable.

\section{Competing interests}

The authors declare that they have no competing interests.

\section{Publisher's Note}

Springer Nature remains neutral with regard to jurisdictional claims in published maps and institutional affiliations.

\section{Author details}

'Department of Urology, Renji Hospital, School of Medicine in Shanghai Jiao Tong University, 160 Pujian Road, Pudong District, Shanghai 200127, China. ${ }^{2}$ Department of Urology, Shanghai Tenth People's Hospital, Nanjing Medical University, Nanjing 211166, China. ${ }^{3}$ Department of Urology, Shanghai Tenth People's Hospital, School of Medicine in Tongji University, Shanghai 200072, China. ${ }^{4}$ Department of Urology, Shanghai First People's Hospital, School of Medicine in Shanghai Jiao Tong University, Shanghai 200080, China.

Received: 12 May 2018 Accepted: 15 October 2018

Published online: 12 November 2018

\section{References}

1. Torre LA, Bray F, Siegel RL, Ferlay J, Lortet-Tieulent J, Jemal A. Global cancer statistics, 2012. CA Cancer J Clin. 2015;65:87-108. https://doi.org/10.3322/ caac. 21262 .

2. Jonasch E, Gao J, Rathmell WK. Renal cell carcinoma. BMJ. 2014:349:g4797.

3. Chen C, Xue S, Zhang J, Chen W, Gong D, Zheng J, Ma J, Xue W, Chen Y, Zhai $W$, Zheng J. DNA-methylation-mediated repression of miR-766-3p promotes cell proliferation via targeting SF2 expression in renal cell carcinoma. Int J Cancer. 2017;141:1867-78. https://doi.org/10.1002/ijc.30853.

4. Buti S, Bersanelli M, Sikokis A, Maines F, Facchinetti F, Bria E, Ardizzoni A, Tortora G, Massari F. Chemotherapy in metastatic renal cell carcinoma today? A systematic review. Anti-Cancer Drugs. 2013;24:535-54. https://doi. org/10.1097/CAD.0b013e3283609ec1.

5. Ljungberg B, Bensalah K, Canfield S, Dabestani S, Hofmann F, Hora M, Kuczyk MA, Lam T, Marconi L, Merseburger AS, et al. EAU guidelines on renal cell carcinoma: 2014 update. Eur Urol. 2015;67:913-24. https://doi.org/ 10.1016/j.eururo.2015.01.005.

6. Mohr AM, Mott JL. Overview of microRNA biology. Semin Liver Dis. 2015;35: 3-11. https://doi.org/10.1055/s-0034-1397344.

7. Hammond SM. An overview of microRNAs. Adv Drug Deliv Rev. 2015;87:314. https://doi.org/10.1016/j.addr.2015.05.001.

8. Tan W, Liu B, Qu S, Liang G, Luo W, Gong C. MicroRNAs and cancer: key paradigms in molecular therapy. Oncol Lett. 2018;15:2735-42. https://doi. org/10.3892/ol.2017.7638.

9. Drusco A, Croce CM. MicroRNAs and Cancer: a long story for short RNAs. Adv Cancer Res. 2017;135:1-24. https://doi.org/10.1016/bs.acr.2017.06.005.

10. Li X, Liu X, Xu W, Zhou P, Gao P, Jiang S, Lobie PE, Zhu T. c-MYC-regulated miR-23a/24-2/27a cluster promotes mammary carcinoma cell invasion and hepatic metastasis by targeting Sprouty2. J Biol Chem. 2013;288:18121-33. https://doi.org/10.1074/jbc.M113.478560.

11. Chen X, Wang X, Ruan A, Han W, Zhao Y, Lu X, Xiao P, Shi H, Wang R, Chen $L$, et al. miR-141 is a key regulator of renal cell carcinoma proliferation and metastasis by controlling EphA2 expression. Clin Cancer Res. 2014;20:261730. https://doi.org/10.1158/1078-0432.CCR-13-3224.

12. Lei L, Huang Y, Gong W. miR-205 promotes the growth, metastasis and chemoresistance of NSCLC cells by targeting PTEN. Oncol Rep. 2013;30: 2897-902. https://doi.org/10.3892/or.2013.2755.

13. Li W, Liu M, Feng Y, Xu YF, Huang YF, Che JP, Wang GC, Yao XD, Zheng JH. Downregulated miR-646 in clear cell renal carcinoma correlated with tumour metastasis by targeting the Nin one binding protein (NOB1). Br J Cancer. 2014;111:1188-200. https://doi.org/10.1038/bjc.2014.382. 
14. Li RZ, Wang LM. Decreased microRNA-452 expression and its prognostic significance in human osteosarcoma. World J Surg Oncol. 2016;14:150. https://doi.org/10.1186/s12957-016-0900-y.

15. Hu Q, Chen WX, Zhong SL, Zhang JY, Ma TF, Ji H, Lv MM, Tang JH, Zhao JH. MicroRNA-452 contributes to the docetaxel resistance of breast cancer cells. Tumour Biol. 2014;35:6327-34. https://doi.org/10.1007/s13277-014-1834-z.

16. Zhang $Y$, Han L, Pang J, Wang Y, Feng F, Jiang Q. Expression of microRNA452 via adenoviral vector inhibits non-small cell lung cancer cells proliferation and metastasis. Tumour Biol. 2016;37:8259-70. https://doi.org/ 10.1007/s13277-015-4725-Z.

17. Zheng Q, Sheng Q, Jiang C, Shu J, Chen J, Nie Z, Lv Z, Zhang Y. MicroRNA452 promotes tumorigenesis in hepatocellular carcinoma by targeting cyclin-dependent kinase inhibitor 1B. Mol Cell Biochem. 2014;389:187-95. https://doi.org/10.1007/s11010-013-1940-z.

18. Goto Y, Kojima S, Kurozumi A, Kato M, Okato A, Matsushita R, Ichikawa T, Seki N. Regulation of E3 ubiquitin ligase-1 (WWP1) by microRNA-452 inhibits cancer cell migration and invasion in prostate cancer. Br J Cancer. 2016;114: 1135-44. https://doi.org/10.1038/bjc.2016.95.

19. Da SR, Gustafson D, Nogueira L, Werahera PN, Molina WR, Kim FJ. Targeted therapy for metastatic renal carcinoma: an update. J Kidney Cancer VHL. 2014;1:63-73. https://doi.org/10.15586/jkcvhl.2014.14.

20. Mattei J, Da SR, Sehrt D, Molina WR, Kim FJ. Targeted therapy in metastatic renal carcinoma. Cancer Lett. 2014;343:156-60. https://doi.org/10.1016/j. canlet.2013.09.038

21. Conti A, Santoni M, Amantini C, Burattini L, Berardi R, Santoni G, Cascinu S, Muzzonigro G. Progress of molecular targeted therapies for advanced renal cell carcinoma. Biomed Res Int. 2013;2013:419176. https://doi.org/10.1155/ 2013/419176.

22. Ornstein MC, Wood LS, Elson P, Allman KD, Beach J, Martin A, Zanick BR, Grivas P, Gilligan T, Garcia JA, Rini BI. A phase II study of intermittent Sunitinib in previously untreated patients with metastatic renal cell carcinoma. J Clin Oncol. 2017:35:1764-9. https://doi.org/10.1200/JCO.2016.71.1184.

23. Parekh $\mathrm{H}$, Rini Bl. Emerging therapeutic approaches in renal cell carcinoma. Expert Rev Anticancer Ther. 2015;15:1305-14. https://doi.org/10.1586/ 14737140.2015.1090315.

24. Zhai W, Sun Y, Guo C, Hu G, Wang M, Zheng J, Lin W, Huang Q, Li G, Zheng $J$, Chang C. LnCRNA-SARCC suppresses renal cell carcinoma (RCC) progression via altering the androgen receptor(AR)/miRNA-143-3p signals. Cell Death Differ. 2017;24:1502-17. https://doi.org/10.1038/cdd.2017.74.

25. Wang M, Sun Y, Xu J, Lu J, Wang K, Yang DR, Yang G, Li G, Chang C. Preclinical studies using miR-32-5p to suppress clear cell renal cell carcinoma metastasis via altering the miR-32-5p/TR4/HGF/met signaling. Int J Cancer. 2018. https://doi.org/10.1002/ijc.31289.

26. Vermeulen L, De Wilde G, Notebaert S, Vanden BW, Haegeman G. Regulation of the transcriptional activity of the nuclear factor-kappaB p65 subunit. Biochem Pharmacol. 2002;64:963-70.

27. Sun B, Chen L, Fu H, Guo L, Guo H, Zhang N. Upregulation of RICTOR gene transcription by the proinflammatory cytokines through NF-kappaB pathway contributes to the metastasis of renal cell carcinoma. Tumour Biol. 2016;37: 4457-66. https://doi.org/10.1007/s13277-015-4296-z.

28. Meng XM, Huang XR, Xiao J, Chung AC, Qin W, Chen HY, Lan HY Disruption of Smad4 impairs TGF-beta/Smad3 and Smad7 transcriptional regulation during renal inflammation and fibrosis in vivo and in vitro. Kidney Int. 2012;81:266-79. https://doi.org/10.1038/ki.2011.327.

29. Hu Q, Gong JP, Li J, Zhong SL, Chen WX, Zhang JY, Ma TF, Ji H, Lv MM, Zhao $J$, Tang JH. Down-regulation of miRNA-452 is associated with adriamycinresistance in breast cancer cells. Asian Pac J Cancer Prev. 2014;15:5137-42.

30. Rezapour S, Bahrami T, Hashemzadeh S, Estiar MA, Nemati M, Ravanbakhsh R, Feizi MA, Kafil HS, Pouladi N, Ghojazadeh M, Sakhinia E. STC1 and NFkappaB p65 (Rel A) is constitutively activated in colorectal Cancer. Clin Lab. 2016;62:463-9

31. Ku T, Li B, Gao R, Zhang Y, Yan W, Ji X, Li G, Sang N. NF-kappaB-regulated microRNA-574-5p underlies synaptic and cognitive impairment in response to atmospheric PM2.5 aspiration. Part Fibre Toxicol. 2017:14:34. https://doi. org/10.1186/s12989-017-0215-3

32. de la Rica L, Garcia-Gomez A, Comet NR, Rodriquez-Ubreva J, Ciudad L, Vento-Tormo R, Company C, Alvarez-Errico D, Garcia M, Gomez-Vaquero C, Ballestar E. NF-kappaB-direct activation of microRNAs with repressive effects on monocyte-specific genes is critical for osteoclast differentiation. Genome Biol. 2015;16:2. https://doi.org/10.1186/s13059-014-0561-5.
33. Zhou R, Hu G, Liu J, Gong AY, Drescher KM, Chen XM. NF-kappaB p65dependent transactivation of miRNA genes following Cryptosporidium parvum infection stimulates epithelial cell immune responses. PLoS Pathog. 2009:5:e1000681. https://doi.org/10.1371/journal.ppat.1000681.

34. Yang D, Sun C, Zhang J, Lin S, Zhao L, Wang L, Lin R, LV J, Xin S. Proliferation of vascular smooth muscle cells under inflammation is regulated by NF-kappaB p65/microRNA-17/RB pathway activation. Int J Mol Med. 2018;41:43-50. https://doi.org/10.3892/ijmm.2017.3212.

35. Li J, Wang K, Chen X, Meng H, Song M, Wang Y, Xu X, Bai Y. Transcriptional activation of microRNA-34a by NF-kappa B in human esophageal cancer cells. BMC Mol Biol. 2012;13:4. https://doi.org/10.1186/1471-2199-13-4.

36. Li Y, Dai D, Lu Q, Fei M, Li M, Wu X. Sirt2 suppresses glioma cell growth through targeting NF-kappaB-miR-21 axis. Biochem Biophys Res Commun. 2013;441:661-7. https://doi.org/10.1016/j.bbrc.2013.10.077.

37. Zhao M, Mishra L, Deng CX. The role of TGF-beta/SMAD4 signaling in cancer. Int J Biol Sci. 2018;14:111-23. https://doi.org/10.7150/ijbs.23230.

38. Miyaki M, Kuroki T. Role of Smad4 (DPC4) inactivation in human cancer. Biochem Biophys Res Commun. 2003;306:799-804.

39. Mao S, Lu G, Lan X, Yuan C, Jiang W, Chen Y, Jin X, Xia Q. Valproic acid inhibits epithelialmesenchymal transition in renal cell carcinoma by decreasing SMAD4 expression. Mol Med Rep. 2017;16:6190-9. https://doi. org/10.3892/mmr.2017.7394.

40. Xia X, Wu W, Huang C, Cen G, Jiang T, Cao J, Huang K, Qiu. SMAD4 and its role in pancreatic cancer. Tumour Biol 2015, 36:111-119. doi: https://doi.org/ 10.1007/s13277-014-2883-z.

41. Izumi S, Nakamura S, Mano S, Akaki S. Well differentiation and intact Smad4 expression are specific features of groove pancreatic ductal adenocarcinomas. Pancreas. 2015:44:394-400. https://doi.org/10.1097/MPA.0000000000000260.

42. Liu Y, Xu Y, Li X, Chen Z. Smad4 suppresses the progression of renal cell carcinoma via the activation of forkhead box protein $\mathrm{H}$ 1. Mol Med Rep. 2015;11:2717-22. https://doi.org/10.3892/mmr.2014.3061.

43. Wang JY, Gao YB, Zhang N, Zou DW, Wang P, Zhu ZY, Li JY, Zhou SN, Wang SC, Wang YY, Yang JK. miR-21 overexpression enhances TGF-beta1-induced epithelial-to-mesenchymal transition by target smad7 and aggravates renal damage in diabetic nephropathy. Mol Cell Endocrinol. 2014;392:163-72 https://doi.org/10.1016/j.mce.2014.05.018.

44. Smith AL, Iwanaga R, Drasin DJ, Micalizzi DS, Vartuli RL, Tan AC, Ford HL. The miR-106b-25 cluster targets Smad7, activates TGF-beta signaling, and induces EMT and tumor initiating cell characteristics downstream of Six 1 in human breast cancer. Oncogene. 2012;31:5162-71. https://doi.org/10.1038/ onc.2012.11.

45. Xia H, Ooi LL, Hui KM. MicroRNA-216a/217-induced epithelial-mesenchymal transition targets PTEN and SMAD7 to promote drug resistance and recurrence of liver cancer. Hepatology. 2013;58:629-41. https://doi.org/10.1002/hep.26369.

46. Passadouro M, Pedroso DLM, Faneca H. MicroRNA modulation combined with sunitinib as a novel therapeutic strategy for pancreatic cancer. Int J Nanomedicine. 2014;9:3203-17. https://doi.org/10.2147/IJN.S64456.

47. Goto Y, Kurozumi A, Nohata N, Kojima S, Matsushita R, Yoshino H, Yamazaki K, Ishida Y, Ichikawa T, Naya Y, Seki N. The microRNA signature of patients with sunitinib failure: regulation of UHRF1 pathways by microRNA-101 in renal cell carcinoma. Oncotarget. 2016;7:59070-86. https://doi.org/10.18632/ oncotarget.10887.

48. Khella H, Butz H, Ding Q, Rotondo F, Evans KR, Kupchak P, Dharsee M, Latif A, Pasic MD, Lianidou $E$, et al. miR-221/222 are involved in response to Sunitinib treatment in metastatic renal cell carcinoma. Mol Ther. 2015;23: 1748-58. https://doi.org/10.1038/mt.2015.129.

49. Cunha PP, Costa PM, Morais CM, Lopes IR, Cardoso AM, Cardoso AL, Mano M, Jurado AS, Pedroso DLM. High-throughput screening uncovers miRNAs enhancing glioblastoma cell susceptibility to tyrosine kinase inhibitors. Hum Mol Genet. 2017;26:4375-87. https://doi.org/10.1093/hmg/ddx323.

50. Shin HW, Cho CH, Kim TY, Park JW. Sunitinib deregulates tumor adaptation to hypoxia by inhibiting HIF-1alpha synthesis in HT-29 colon cancer cells. Biochem Biophys Res Commun. 2010;398:205-11. https://doi.org/10.1016/j. bbrc.2010.06.060. 